\title{
The conical conformal MEMS quasi-end-fire array antenna
}

\author{
Lin Cong*, Lixin Xu, Jianhua Li, Ting Wang and Qi Han \\ School of Mechatronical Engineering, \\ Beijing Institute of Technology, Beijing 100081, China \\ *lincong@bit.edu.cn
}

Received 5 October 2016

Revised 18 February 2017

Accepted 21 February 2017

Published 2 March 2017

\begin{abstract}
The microelectromechanical system (MEMS) quasi-end-fire array antenna based on a liquid crystal polymer (LCP) substrate is designed and fabricated in this paper. The maximum radiation direction of the antenna tends to the cone axis forming an angle less than $90^{\circ}$, which satisfies the proximity detection system applied at the forward target detection. Furthermore, the proposed antenna is fed at the ended side in order to save internal space. Moreover, the proposed antenna takes small covering area of the proximity detection system. The proposed antenna is fabricated by using the flexible MEMS process, and the measurement results agree well with the simulation results. This is the first time that a conical conformal array antenna is fabricated by the flexible MEMS process to realize the quasi-end-fire radiation. A pair of conformal MEMS array antennas resonates at $14.2 \mathrm{GHz}$ with its mainlobes tending to the cone axis forming a $30^{\circ}$ angle and a $31^{\circ}$ angle separately, and the gains achieved are $1.82 \mathrm{~dB}$ in two directions, respectively. The proposed antenna meets the performance requirements for the proximity detection system which has vast application prospects.
\end{abstract}

Keywords: Conical; conformal; MEMS; end-fire; antenna.

\section{Introduction}

With the miniaturization and integration of the modern communication system, the installation space of the antenna is usually limited, $\frac{1}{-}$ such as in flying systems, $\stackrel{2}{-}-\underline{4}$ mobile communication systems ${ }^{5}$ and automotive systems..$\underline{-6}$ The conformal antenna could be mounted on the platform surface to solve the above problem reaching the purpose of saving its internal space. Furthermore, conformal antennas are employed

* Corresponding author.

This is an Open Access article published by World Scientific Publishing Company. It is distributed under the terms of the Creative Commons Attribution 4.0 (CC-BY) License. Further distribution of this work is permitted, provided the original work is properly cited. 


\section{Cong et al.}

on different types of surfaces, which depends on the geometry of the communication system.

In the international literature one can find several studies of conical, cylindrical and spherical conformal arrays useful for omnidirectional as well as directional applications. $\frac{9}{}$ The study of this area over the last decades has resulted in various antenna designs in literature. Marulier presented a conical wraparound short-circuited microstrip antenna operating in dual frequency ranges with the beam peak oriented along $75^{\circ}$ from the cone axis. $\frac{10}{}$ Wang et al. designed a millimeter wave cylindrical conformal array with conical beams having their maximum radiation at about $32^{\circ}$ from the axis of the cylinder. $\frac{11}{}$ Xuan proposed a conical conformal antenna array leading to an omnidirectional broadside pattern. $\frac{12}{}$ Bernard presented the radiation pattern of a monopole antenna mounted on the projectile with its main beam at $34^{\circ}$ towards the projectile back..$^{13}$ Lin et al. designed a cylindrical microstrip leakywave antenna with its radiation pattern scanning from $33^{\circ}$ to $67^{\circ}$ by varying the frequency from $10 \mathrm{GHz}$ to $12 \mathrm{GHz} \cdot \frac{14}{}$ It is worth noting that conformal antennas can realize the directional radiation with a certain tilted beam. However, the leakywave antenna takes large surface area of the platform, the back-fed antenna takes large interior space of the platform and the short-circuited microstrip antenna with an annular slot requires complex processes in the manufacture. Therefore, small covering area, small occupying space and simplified processes are both challenges and development prospects.

The conical shape surface can be of special interest for applications in the noses of missile, aircraft and instrument. ${ }^{15}$ Moreover, the radiation of the conical conformal arrays has been studied in Refs. $16-20$. In this context, an array antenna conforming to a cone surface is supposed to be applied to the forward target detection system at around $14 \mathrm{GHz}$. In the forward target detection system, the radiation pattern of its conformal antenna should exhibit specific characteristics including radiating a tilted beam on the vertical plane.

The conical conformal antenna in this paper is applied to the proximity detection system of the projectile. The proximity detection system is located at the head of the projectile. The usable distribution space for the antenna is the front end of projectile head. Therefore the design of conformal antenna mounted on the projectile surface saves the internal space of the projectile. The proximity detection system consists of the antenna, the radio-frequency signal processing circuit, the intermediate-frequency signal processing circuit and the digital signal processing circuit. Moreover, in the proximity detection system, wireless links can be established dynamically to transmit data from the projectile to the target. As the very front-end component of the proximity detection system, the proposed antenna is used for directly coupling electromagnetic environment with the outside world. The proximity detection system of the projectile needs the antenna to radiate forward along the projectile head, and the antenna effects are better with the radiation direction tending closer to the cone axis. Thus this paper proposed a demand to maintain the forward radiation, which is the direction of maximum radiation at 
an angle less than $90^{\circ}$ to the cone axis, and $90^{\circ}$ is the half-angle of the radiation range.

The $14 \mathrm{GHz}$ is chosen as the central frequency of the antenna because it makes the antenna small and suitable for the application of small space. Furthermore, the $14 \mathrm{GHz}$ central frequency of the antenna is the carrier frequency of the proximity detection system. It is worth noting that the distance resolution is decided by the ranging algorithm, and not by the carrier frequency of the proximity detection system. The proximity detection system in this paper adopts the frequency modulation continuous wave (FMCW) ranging method. In the frequency modulation ranging method, typical ranging algorithms include basic fast Fourier transform $(\mathrm{FFT})^{21}$ and zero-padded FFT. ${ }^{22,23}$ In the basic FFT ranging algorithm, ${ }^{21}$ the distance resolution is derived from the modulated frequency deviation. By setting the modulated frequency deviation as $100 \mathrm{MHz}$, the corresponding distance resolution of $1.5 \mathrm{~m}$ is achieved. In the zero-padded FFT ranging algorithm, ${ }^{22}$ the distance resolution is derived from the number of zero-padded circles and the modulated frequency deviation. By setting the number of zero-padded circles as 5 and the modulated frequency deviation as $10 \mathrm{MHz}$, the corresponding distance resolution of $1.5 \mathrm{~m}$ is achieved. Such distance resolution of $1.5 \mathrm{~m}$ meets the requirement of the proximity detection system. A finer distance resolution can be obtained by changing the number of zero-padded circles and the modulated frequency deviation.

As is well known, microstrip antennas are preferably conformal to the host surface, allowing an integrated nonprotruding solution to provide good radiation coverage. ${ }^{24}$ Microstrip antennas have the advantages of low weight, thin profile and easy fabrication, but suffer from a relatively narrow bandwidth and limitations on maximum transmit power. Nevertheless such constraints are acceptable in the application of this paper since priority is given to conformability. ${ }^{2}$ Therefore, the microstrip antenna was chosen as the unit of the proposed array antenna in this paper.

The silicon micromachining has long been the mainstream of microelectromechanical system (MEMS) technology because of its maturity and its strong capability of processing. However the brittleness of silicon affects its application range. Flexible electronics technology on substrates of flexible plastics and thin metals emerges owing to its unique malleability and efficient manufacturing process, and it is applicable to conformal fields. $\frac{25}{}$ A number of polymer materials have been applied to MEMS recently, in which liquid crystal polymer (LCP) is used in MEMS successfully and widely as shown in Refs. 26-29. Hence, the flexible MEMS technology was a good choice for making conformal antennas in this paper.

In this paper, a quasi-end-fire microstrip array antenna with flexible MEMS technology conforming to a conical surface was first designed and fabricated. The proposed antenna dealt with the array layout to archive certain tilted mainlobes in its radiation, and it can be applied at the forward target detection. After the introduction, the Sec. 2 describes the antenna design and its simulation of radiation performance in the planar and conical conformal states. The fabrication 


\section{Cong et al.}

and measurement results are provided in Sec. 3 and conclusions are drawn in Sec. 4 .

\section{Antenna Design and Simulation}

The ordinary end-fire array of antenna is a linear array with its maximum radiation pointing along the direction of array layout. ${ }^{30}$ Therefore, the ordinary end-fire array theory was adopted to design the antenna to make the direction of maximum radiation at an angle to its carrier axis. According to the ordinary end-fire array theory, the length of the visible space is described by

$$
2 k d \leq 2 \pi-\frac{\pi}{N}
$$

where $k=2 \pi / \lambda$, thus the spacing between the antenna units is achieved by

$$
d \leq \frac{\lambda}{2}\left(1-\frac{1}{2 N}\right),
$$

in which $\lambda$ is the wavelength in the LCP substrate at the resonant frequency. Correspondingly, the stepped value of phase satisfying

$$
\alpha=k d
$$

has a single mainlobe at $\theta=180^{\circ}$ forming the end-fire radiation.

The MEMS array antenna is composed of two units in order to take small covering area, so the spacing between two adjacent units was $d \leq 3 \lambda / 8$ got from Eq. (2) when $N=2$. Moreover, the stepped value of phase $\alpha=3 \pi / 4$ of the proposed antenna was obtained by calculating from Eq. (3) when $d=3 \lambda / 8$. Such values are used in the antenna design.

\subsection{The MEMS array antenna in planar state}

The proposed antenna was fabricated by using the flexible micromachining process on an LCP substrate which is a flexible high-molecule polymer material with a thickness of $100 \mu \mathrm{m}$ and a relative permittivity of 3.14. The simulations of the antenna were carried out by using HFSS, and the design variables have been optimized at $14 \mathrm{GHz}$. The schematic of the MEMS array antenna is shown in Fig. 1, and its geometrical dimension is $25 \times 15 \mathrm{~mm}^{2}$. The structure parameters of the MEMS array antenna are summarized in Table 1.

The return loss of the MEMS array antenna in planar state is illustrated in Fig. 2. It can be seen the antenna resonates at a frequency of $14 \mathrm{GHz}$ and exhibits a return loss of $-29 \mathrm{~dB}$. Furthermore, the $-10 \mathrm{~dB}$ return loss bandwidth is $99 \mathrm{MHz}$.

The radiation patterns of the MEMS array antenna in planar state are presented in Fig. 3. As depicted by the three-dimensional (3D) radiation pattern in Fig. 3(a), the gain at the resonant frequency is $7.4 \mathrm{~dB}$. Owing to the microstrip antenna having high gain and low half-power beam width relatively, the spacing between the antenna elements is calculated from the edge of the patch. Such calculation 


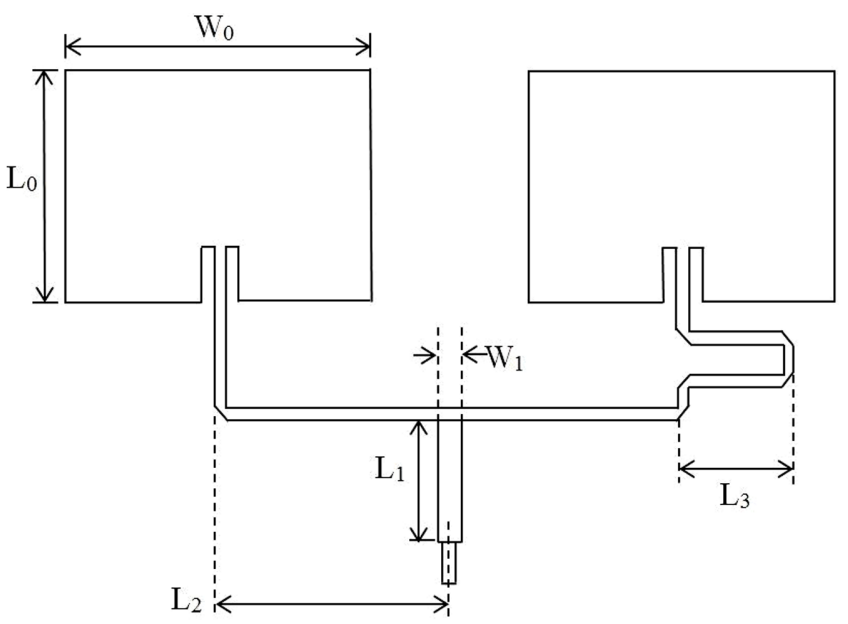

Fig. 1. Schematic of the MEMS array antenna.

Table 1. Structure parameters of the MEMS array antenna.

\begin{tabular}{cc}
\hline Parameters & Value $(\mathrm{mm})$ \\
\hline$L_{0}$ & 6.008 \\
$L_{1}$ & 3.3 \\
$L_{2}$ & 6.135 \\
$L_{3}$ & 2.945 \\
$W_{0}$ & 7.446 \\
$W_{1}$ & 0.395 \\
\hline
\end{tabular}

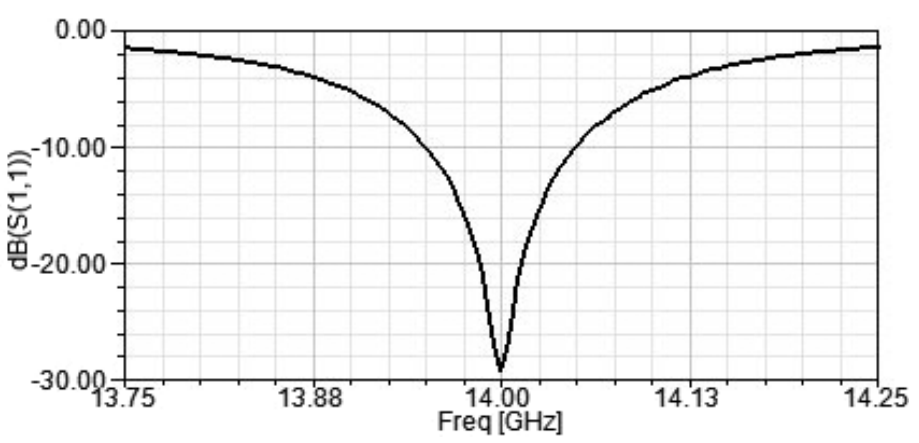

Fig. 2. Return loss of the MEMS array antenna in planar state.

method was used in the simulation leading to the mainlobe tending to the direction of array layout forming an angle. As shown in Fig. 3(b), it can be seen in the $H$ plane radiation that the mainlobe direction is $\theta=28^{\circ}$. Thus the mainlobe tends to the direction of array layout forming $62^{\circ}$, such an angle is larger than the end-fire angle which is because of the reflection effect of the ground plate. 


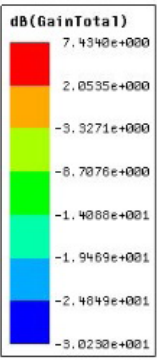

(a) $3 \mathrm{D}$ radiation pattern

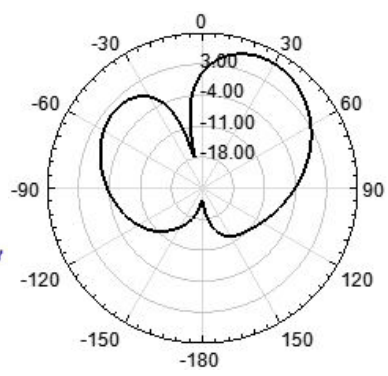

(b) $H$-plane

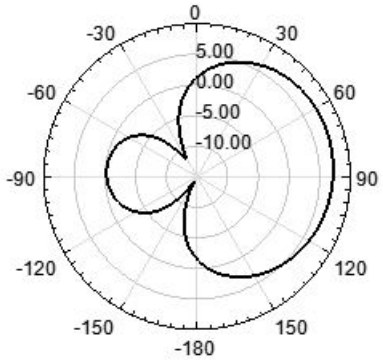

(c) E-plane

Fig. 3. (Color online) Radiation patterns of the MEMS array antenna in planar state.

\subsection{One conformal MEMS array antenna}

The shape of the forward target detection system is a truncated cone with a base radius of $12.5 \mathrm{~mm}$ and a height of $24 \mathrm{~mm}$. The material of the cone was performed as a perfectly electric conductor using HFSS. Besides, the MEMS array antenna is conformal to the surface of the cone, and the arrangement line of the array is pointing to the vertex of the cone, as shown in Fig. 4.

We studied the radiation characteristics of one conformal MEMS array antenna. Its return loss is represented in Fig. 5. It can be seen that the center frequency is 14.02 $\mathrm{GHz}$ with its return loss of $-24.4 \mathrm{~dB}$, and the bandwidth is $85 \mathrm{MHz}$ when the return loss is less than $-10 \mathrm{~dB}$.

The radiation patterns of one conformal MEMS array antenna are plotted in Fig. 6. As shown in Fig. 6(a), the gain at the center frequency is $3.5 \mathrm{~dB}$. Since the $H$-plane radiation pattern presents that the mainlobe direction is $\theta=54^{\circ}$ as shown in Fig. 6(b), the main beam is toward the cone axis with a $36^{\circ}$ tilt. Compared

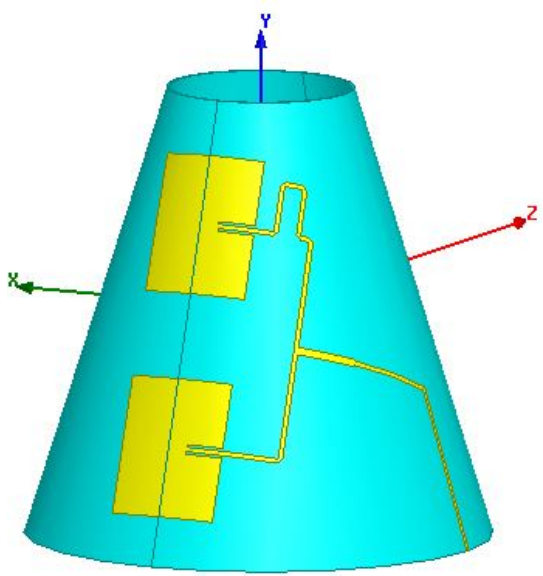

Fig. 4. Side view of one conformal MEMS array antenna. 




Fig. 5. Return loss of one conformal MEMS array antenna.

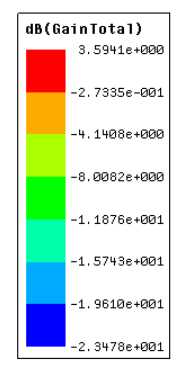

(a) $3 \mathrm{D}$ radiation pattern

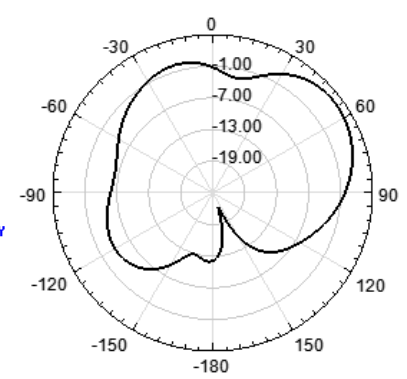

(b) $H$-plane



(c) E-plane

Fig. 6. (Color online) Radiation patterns of one conformal MEMS array antenna.

with the planar MEMS array antenna, a slight shift of the resonant frequency towards a higher value can be observed. This variation can be attributed to the effective spacing between two radiation edges of the patch, which is shorter for the conformal antenna. Moreover, the return loss increases as the MEMS array antenna becomes conformal to the cone, which indicates the impedance matching becomes worse. However, the return loss is still far away below $-10 \mathrm{~dB}$. Additionally, the bandwidth of the conformal antenna is a little narrower than that of the planar one. Furthermore, by comparison with two-dimensional (2D) radiation patterns of the planar antenna, we found that the lobe width of the conformal antenna becomes broader and its gain becomes lower.

\subsection{A pair of conformal MEMS array antennas}

As shown in Fig. 7, the array antenna is composed of two subarrays. Such a pair of MEMS array antennas is conformal to the surface of the cone in order to obtain symmetrical radiation ranges.

The return loss of a pair of conformal MEMS array antennas is illustrated in Fig. 8. It can be seen that a pair of conformal MEMS array antennas resonates at a 


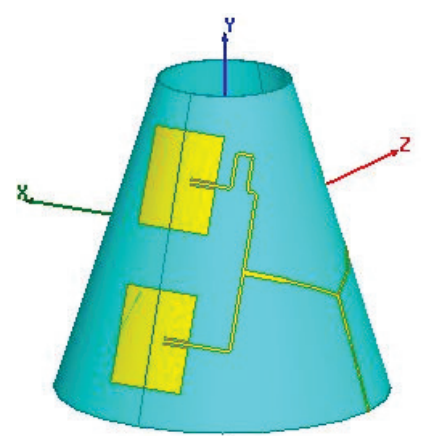

(a) $3 \mathrm{D}$ view of the cone

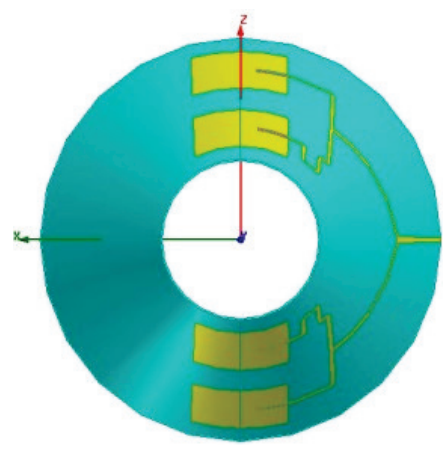

(b) Top view of the cone

Fig. 7. Model of a pair of conformal MEMS array antennas.

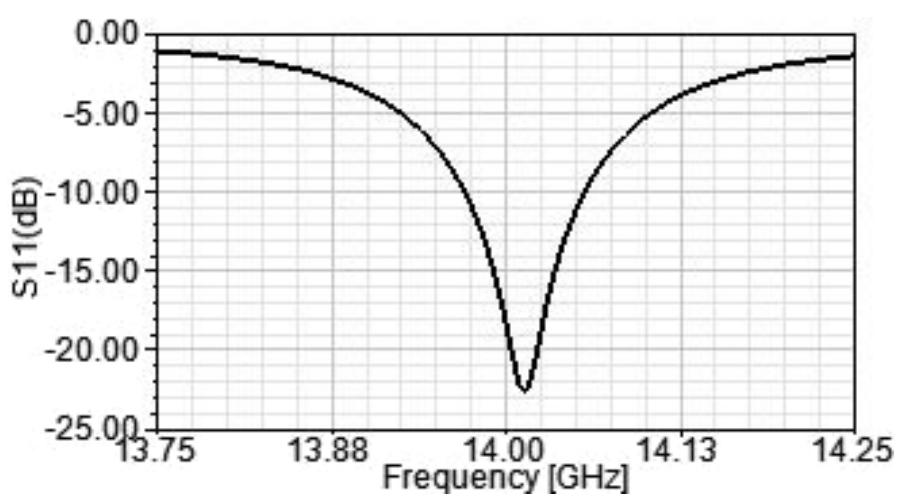

Fig. 8. Return loss of a pair of conformal MEMS array antennas.

frequency of $14.02 \mathrm{GHz}$ and exhibits a return loss of $-22.4 \mathrm{~dB}$, and the bandwidth at $-10 \mathrm{~dB}$ return loss is $85 \mathrm{MHz}$. Apparently the results are almost the same with those of one conformal MEMS array antenna, because the structure dimensions affecting the resonant frequency and the impedance matching are invariant.

The gains at the resonant frequency are $1.84 \mathrm{~dB}$ in two directions, respectively, as presented in Fig. 9(a). A decline can be observed in gains compared with one conformal antenna, which indicates that the mutual coupling of two conformal antennas acts caused by their near spacing. Figures 9(b) and 9(c) display the 2D radiation patterns of a pair of conformal MEMS array antennas. The $H$-plane radiation pattern possesses beam peaks oriented along $\theta=57^{\circ}$ and $\theta=124^{\circ}$, respectively. Correspondingly, the mainlobes of a pair of conformal MEMS array antennas tend to the cone axis forming a $33^{\circ}$ and a $34^{\circ}$ angle separately. 


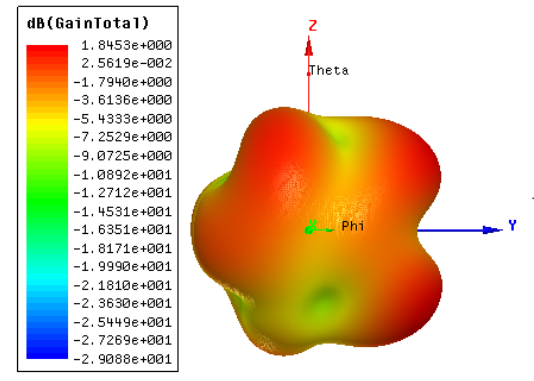

(a) $3 \mathrm{D}$ radiation pattern

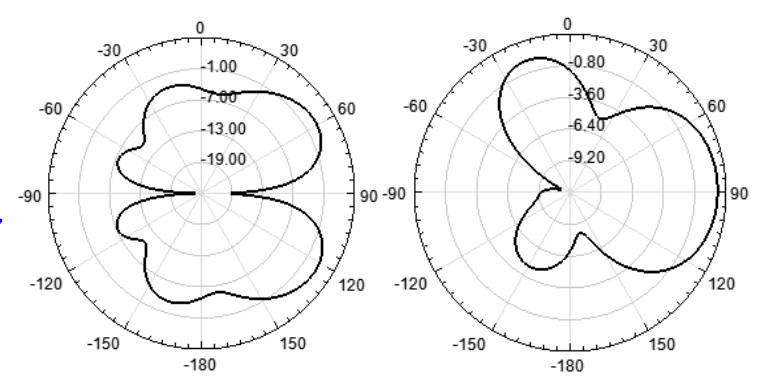

(b) $H$-plane

(c) E-plane

Fig. 9. (Color online) Radiation patterns of a pair of conformal MEMS array antennas.

\section{Fabrication and Measurement Results}

The array antennas were fabricated on a $100-\mu \mathrm{m}$ thick LCP substrate. The LCP substrate used in this paper is the Ultralam 3850HT from Rogers Corporation because of its good electric and mechanical properties. It is a double copper-clad LCP dielectric sheet with each copper layer of thickness $9 \mu \mathrm{m}$. The dielectric properties of the LCP were characterized at $10 \mathrm{GHz}$ to be $\varepsilon_{r}=3.14$ and $\tan \delta=0.002$ reported by the manufacturer.

The flexible MEMS technology was performed to fabricate the array antennas. First of all, the LCP substrate was cleaned using isopropyl alcohol and dried for use. Second, the wafer with size of 4 inches was prepared to be the carrier. Since the LCP substrate is a flexible polymer material leading to the uneven photoresist in the photolithography process, it is necessary to stick it on the wafer to keep its flatness. Third, a 20- $\mu \mathrm{m}$ AZ4903 photoresist was spin-coated on the silicon wafer. Following the spin-coating, the surface mounting process was carried out at room temperature for 5 min to make the LCP substrate adhere to the wafer. Fourth, a $2-\mu$ m RZJ304 resist layer was patterned by photolithography on top of the LCP substrate with the mask defining the antenna shape. Fifth, the copper pattern was realized by chemical etching with a solution of mixed acid which is composed of phosphoric acid, nitric acid and glacial acetic acid. Finally, the LCP substrate was released from wafer into the acetone solution, and then we got the flexible array antennas. Figure 10(a) represents a photograph of the copper pattern when all processes were finished, and Fig. 10(b) shows the flexibility of the MEMS array antenna.

The flexible MEMS array antennas were mounted on the planar and the conical aluminum carriers with conductive adhesive separately. Moreover, the feed line of the array antenna was soldered to a $50-\Omega$ impedance SMA connecter. The return loss measurement was done using an Agilent PNA-E series E8362C vector network analyzer. 


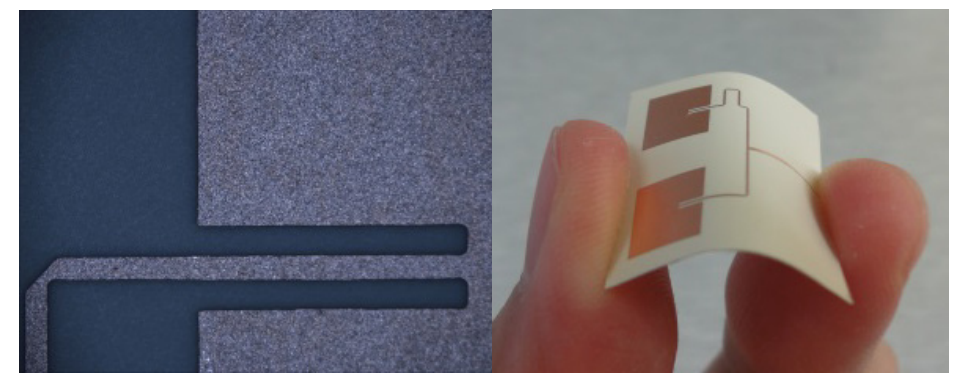

(a) The copper pattern

(b) The MEMS array antenna

Fig. 10. Photographs of the copper pattern and the MEMS array antenna.

\subsection{The MEMS array antenna in planar state}

A photograph of the fabricated MEMS array antenna mounted on a planar carrier is presented in Fig. 11. The return loss measurement of the MEMS array antenna in planar state is shown in Fig. 12. The measured resonant frequency of the antenna was $14.146 \mathrm{GHz}$, which was a little higher than the simulation result, since fabrication tolerance occurs in the processing which leads to a slight discrepancy in the resonant frequency. Besides, the return loss at $14.146 \mathrm{GHz}$ was $-21.6 \mathrm{~dB}$. The bandwidth at $-10 \mathrm{~dB}$ return loss was $15 \mathrm{MHz}$. A higher return loss and a narrow bandwidth can be observed compared with the simulation results; because the matching effect between the transmission line and the coaxial pin is not optimized as the simulation. Moreover, the narrow bandwidth is due to the thin thickness of the dielectric material compared to its wavelength at $14 \mathrm{GHz}$. The measured radiation patterns of the MEMS array antenna in planar state are presented in Fig. 13. As depicted in Fig. 13(a), the gain at the resonant frequency is $7.38 \mathrm{~dB}$, and the mainlobe direction is $\theta=34^{\circ}$. Thus the mainlobe tends to the direction of array layout forming $56^{\circ}$.

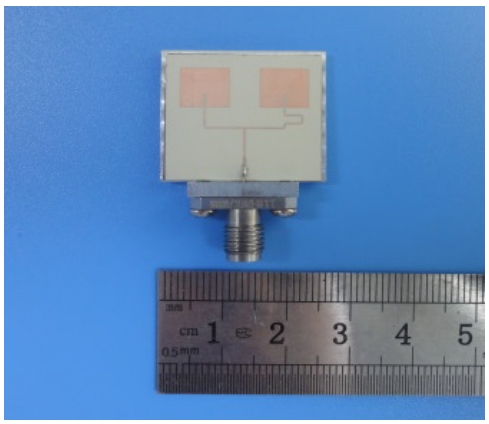

Fig. 11. The fabricated MEMS array antenna mounted on a planar carrier. 


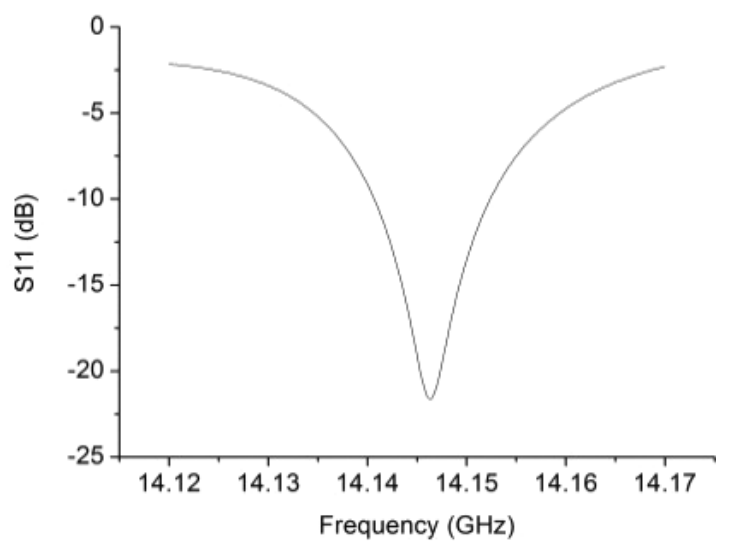

Fig. 12. Return loss of the MEMS array antenna in planar state.

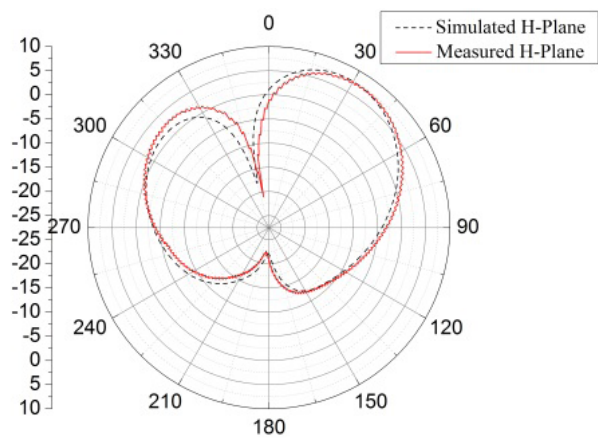

(a) $H$-plane

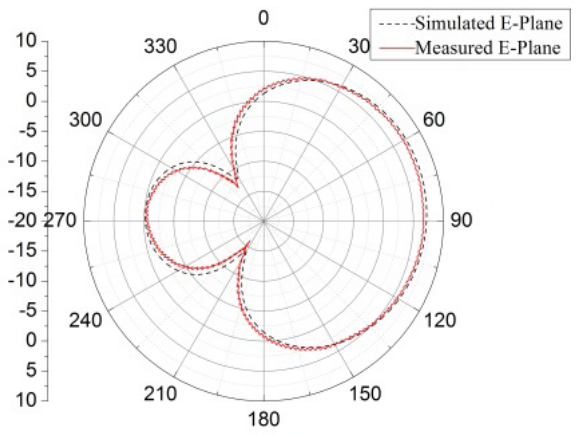

(b) E-plane

Fig. 13. (Color online) Measured radiation patterns of the MEMS array antenna in planar state.

\subsection{One conformal MEMS array antenna}

A photograph of one fabricated conformal MEMS array antenna mounted on a conical carrier is presented in Fig. 14. The measured return loss of one conformal MEMS array antennas is illustrated in Fig. 15. As compared to the simulation result, the conformal antenna had a higher resonant frequency of $14.2 \mathrm{GHz}$ and a higher return loss of $-21.5 \mathrm{~dB}$. Furthermore, the $-10 \mathrm{~dB}$ return loss bandwidth was $13 \mathrm{MHz}$. The return loss is deteriorated a little due to the assembly. Because the fabricated antenna is mounted on an aluminum carrier, it interacts with the aluminum carrier and the connector. We further note that, as compared to the measurement result of the planar MEMS array antenna, the conformal antenna had a higher frequency. This proves that the resonant frequency becomes higher when the effective spacing between two radiation edges of the patch gets shorter. The measured radiation patterns of one conformal MEMS array antenna are plotted in Fig. 16. As shown in Fig. 16(a), the gain at the center frequency is $3.4 \mathrm{~dB}$. 


\section{Cong et al.}

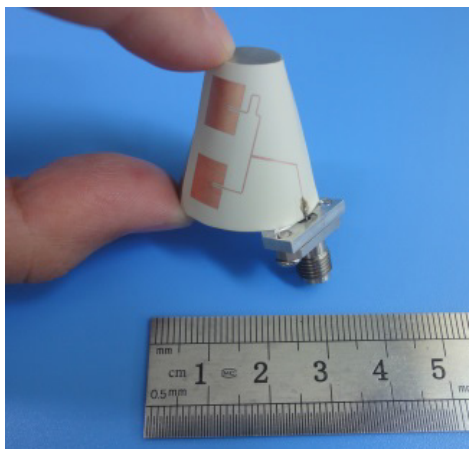

Fig. 14. One fabricated conformal MEMS array antenna mounted on a conical carrier.



Fig. 15. Return loss of one conformal MEMS array antenna.

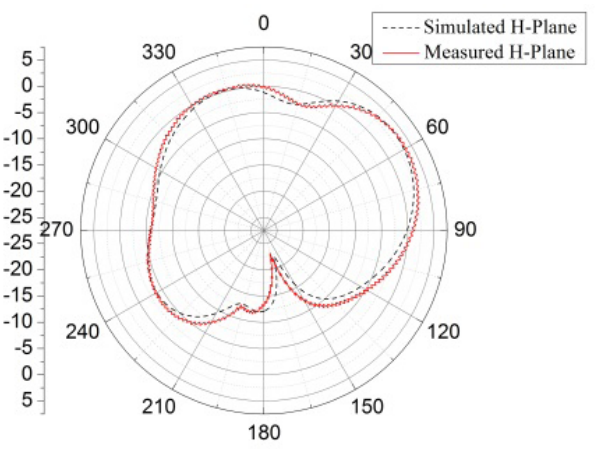

(a) $H$-plane

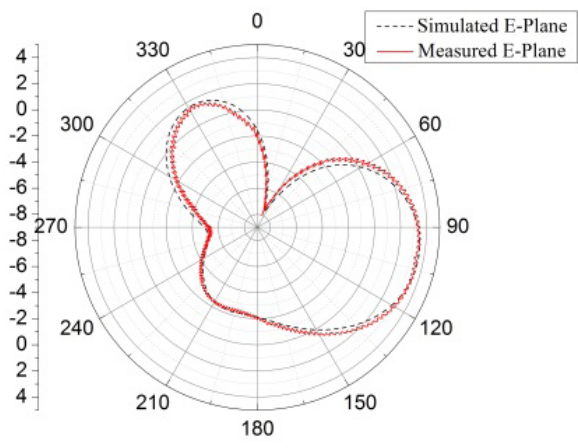

(b) E-plane

Fig. 16. (Color online) Measured radiation patterns of one conformal MEMS array antenna. 
Since the $H$-plane radiation pattern presents that the mainlobe direction is $\theta=59^{\circ}$, the main beam is toward the cone axis with a $31^{\circ}$ tilt.

\subsection{A pair of conformal MEMS array antennas}

Figure 17 shows a photograph of a pair of fabricated conformal MEMS array antennas mounted on a conical carrier. Figure 18 shows the measured return loss of a pair of conformal MEMS array antenna. A pair of conformal MEMS array antennas was resonated at $14.2 \mathrm{GHz}$ with a measured return loss of $-19.2 \mathrm{~dB}$. As expected, the measured result was in good agreement with the measured result of one conformal MEMS array antenna. Such agreement demonstrates the consistency between one conformal MEMS array antenna and a pair of conformal MEMS array antennas which has been shown in simulation results. The bandwidth at $-10 \mathrm{~dB}$ return loss was $13 \mathrm{MHz}$. Because the conductive adhesive was used to glue the copper layer and the aluminum carrier together, bubbles between the ground plane of the antenna and the carrier formed capacitors. Such practical issues in simulation are not taken

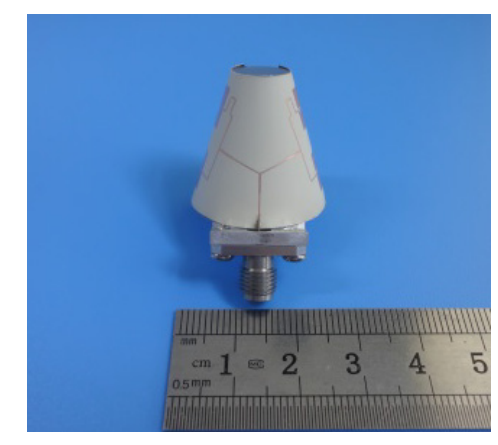

Fig. 17. A pair of fabricated conformal MEMS array antennas mounted on a conical carrier.

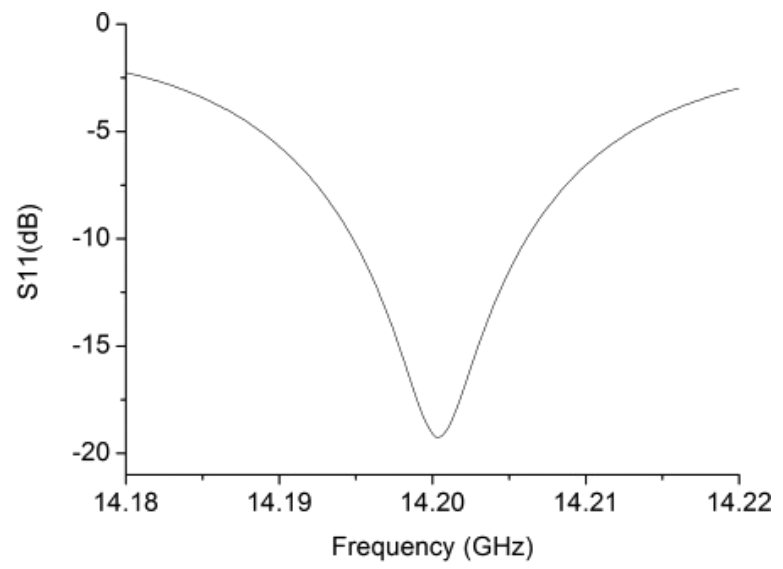

Fig. 18. Return loss of a pair of conformal MEMS array antennas. 


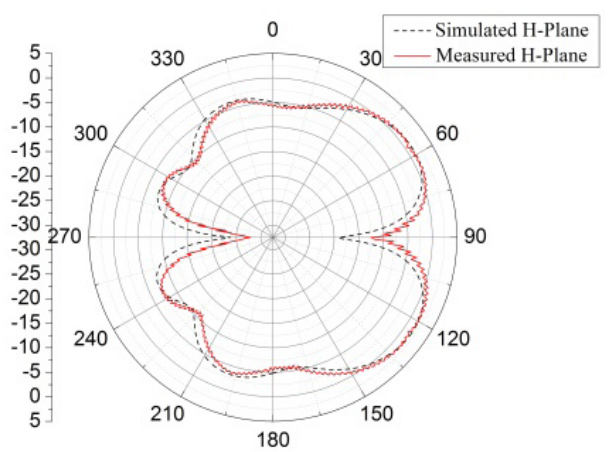

(a) H-plane

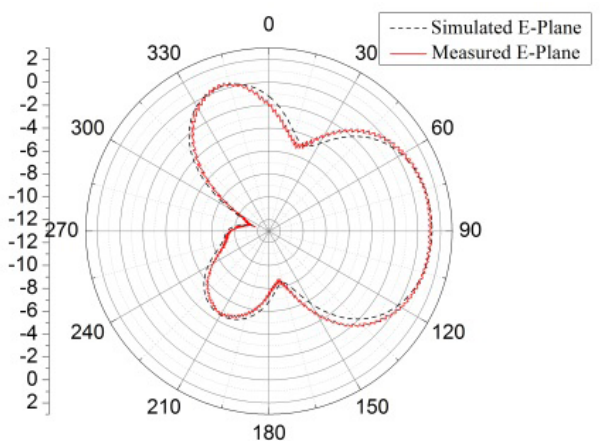

(b) E-plane

Fig. 19. (Color online) Measured radiation patterns of a pair of conformal MEMS array antennas.

into account, which result in a slight mismatch at the input of the antenna in the measurement. The measured radiation patterns of a pair of conformal MEMS array antennas are presented in Fig. 19. The gains at the resonant frequency are $1.82 \mathrm{~dB}$ in two directions, respectively. The $H$-plane radiation pattern possesses beam peaks oriented along $\theta=60^{\circ}$ and $\theta=121^{\circ}$, respectively. Correspondingly, the mainlobes of a pair of conformal MEMS array antennas tend to the cone axis forming a $30^{\circ}$ angle and a $31^{\circ}$ angle separately.

\section{Conclusion}

The conical conformal MEMS quasi-end-fire array antenna was designed and fabricated in this paper. The antenna realized the direction of maximum radiation at an angle less than $90^{\circ}$ to the cone axis. Moreover, the antenna fed at the ended side saved the internal space and had taken small covering area of the proximity detection system. The simulation results of the proposed antenna in the planar and conical conformal states were achieved by using HFSS, and the variations of radiation characteristics from the planar state to the conical conformal state were analyzed. Given the flexible MEMS technology in terms of high precision and simplified processes, the antennas were fabricated and measured. The measurement results were presented and explained to validate the simulation results. This was the first time that a conical conformal array antenna was fabricated by the flexible MEMS process to realize the quasi-end-fire radiation. When a pair of conformal MEMS array antennas is conformal to the cone surface, it resonates at $14.2 \mathrm{GHz}$ with its mainlobes tending to the cone axis forming a $30^{\circ}$ angle and a $31^{\circ}$ angle separately, and the gains achieved are $1.82 \mathrm{~dB}$ in two directions, respectively. The proposed antenna meets the performance requirements for the proximity detection system which has vast application prospects. 


\section{Acknowledgments}

This work was supported by the National Natural Science Foundation of China (Grant No. U1630136).

\section{References}

1. L. Song, in Proc. IEEE 6th Int. Conf. Wireless Communications Networking and Mobile Computing (2010), pp. 1-4.

2. V. Jaeck, L. Bernard, K. Mahdjoubi, R. Sauleau, S. Collardey, P. Pouliguen and P. Potier, IEEE Antennas Wirel. Propag. Lett. 15 (2016) 1230.

3. X. R. Zhou, B. J. Gao, H. D. Wu and Y. H. Ren, Meas. Control Technol. Instrum. 39 (2013) 62.

4. C. Di, H. C. Zhao and Y. X. Yang, J. Detect. Control 36 (2014) 26.

5. A. Y. Svezhentsev, V. Volski, S. Yan, P. J. Soh and G. A. E. Vandenbosch, IEEE Trans. Antennas Propag. 64 (2016) 796.

6. M. Liu, Z. R. Feng and Q. Wu, in Proc. IEEE 2008 China-Japan Joint Microwave Conf. (2008), pp. 121-124.

7. Z. Li, X. Kang, J. Su, Q. Guo, Y. Yang and J. Wang, Int. J. Antennas Propag. 2016 (2016) 1.

8. Y. Zhao and W. Wang, IEEE Antennas Wirel. Propag. Lett. 13 (2014) 1725.

9. X. J. Zhang, X. P. Ma and X. Y. Guan, in Proc. 2009 Int. Conf. Microwave Technology and Computational Electromagnetics (2009), pp. 105-108.

10. O. Marulier, J. Lenormand, J. Saillard and C. Terret, Microw. Opt. Technol. Lett. 13 (1996) 81.

11. H. Wang, D. G. Fang, Y. K. Liu, Y. R. Wang and J. Z. Xu, in Proc. IEEE 2009 Asia Pacific Microwave Conf. (2009), pp. 2010-2013.

12. X. B. Xuan, F. W. Yao and X. Q. Tian, in Proc. IEEE 2013 Int. Symp. Antennas and Propagation (2013), pp. 245-247.

13. L. Bernard, in Proc. IEEE Antennas and Propagation Society Int. Symp. (2008), pp. $1-4$.

14. L. C. Lin, H. Miyagawa, T. Kiatazawa, R. B. Hwang and Y. D. Lin, IEEE Trans. Antennas Propag. 56 (2008) 1853.

15. W. N. Huang, Y. J. Cheng and H. Deng, Int. J. Antennas Propag. 2015 (2015) 1.

16. J. P. Damiano, M. Scotto and J. M. Ribero, Electron. Lett. 32 (1996) 2033.

17. Z. Du, K. Gong, J. S. Fu, Z. Feng and B. Gao, IEEE Trans. Microw. Theory Tech. 48 (2000) 312.

18. R. K. Hersey, W. L. Melvin and J. H. McClellan, Signal Process. 84 (2004) 1481.

19. H. D. Hristov, L. P. Kamburov, R. Feick and J. R. Urumov, Int. J. Infrared Millim. Waves 28 (2007) 451.

20. K. M. Tsui and S. C. Chan, IEEE Trans. Antennas Propag. 58 (2010) 1959.

21. Z. H. Wang, X. Pan and C. Y. Xiang, in Proc. 2012 IEEE 11th Int. Conf. Signal Processing (2012), pp. 1967-1970.

22. G. Q. Qi, in Proc. 2004 IEEE 7th Int. Conf. Signal Processing (2004), pp. 2078-2081.

23. A. J. Hymans and J. Lait, in Proc. IEE B, Electron. Commun. Eng. 107 (1960) 365.

24. J. Ouyang, X. Luo, J. Yang, K. Z. Zhang, J. Zhang and F. Yang, IET Microw. Antennas Propag. 6 (2012) 1277.

25. Z. H. Li, Sci. China Inf. Sci. 12 (2012) 1599.

26. D. J. Chung, D. E. Anagnostou, G. Ponchak, M. Tentzeris and J. Papapolymerou, in Proc. IEEE 2007 Antennas Propagation Society (2007), pp. 93-96. 
L. Cong et al.

27. N. Kingsley, G. E. Ponchak and J. Papapolymerou, IEEE Trans. Antennas Propag. 56 (2008) 108.

28. G. Wang, R. Bairavasubramanian, B. Pan and J. Papapolymerou, IET Microw. Antennas Propag. 5 (2012) 1594.

29. N. Kingsley, E. Anagnostou, M. Tentzeris and J. Papapolymerou, J. Microelectromech. Syst. 16 (2007) 1185.

30. Z. H. Xue, W. M. Li and W. Ren, The Analysis and Synthesis of Antenna Array (Beijing University of Aeronautics and Astronautics Press, Beijing, 2011). 